ARTículo

\title{
¿El retorno de las políticas de desconcentración? Mutaciones en el Programa Nacional de Formación Permanente (PNFP). Córdoba, Argentina
}

\author{
Yanina Débora Maturo ${ }^{a}$
}

María Cecilia Bocchio ${ }^{b}$

\section{Resumen}

En este artículo nos proponemos reconstruir las transformaciones del Programa Nacional de Formación Permanente (PNFP) de profesores denominado "Nuestra Escuela". Centramos el análisis en lo acontecido en la provincia de Córdoba, Argentina, tras el cambio de gobierno, a nivel nacional, en diciembre de 2015. El trabajo de campo se enmarca en dos investigaciones en curso en escuelas secundarias de modalidad técnico profesional y común. Recuperamos aportes de entrevistas realizadas a actores estatales que se desempeñan en diferentes niveles de la administración escolar y que poseen distintas tareas en la puesta en acto del programa. Desde los aportes de la teoría de la regulación social, pretendemos caracterizar las nuevas formas de regulación del programa identificando las estrategias desplegadas por el gobierno central y provincial durante el período 2016-2019.

Palabras clave: Programa Nacional de Formación Permanente. Formación Docente Situada. Políticas Educativas.

\section{Presentación}

En Argentina, la presidencia de Néstor Kirchner marcó el desarrollo de políticas educativas tendientes a fortalecer el sistema de formación docente desde una perspectiva federal. Políticas orientadas tanto a la reforma de la formación docente

\footnotetext{
a Consejo Nacional de Investigaciones Científicas y Técnicas (Conicet), Instituto de Humanidades (IDH), Córdoba, Argentina.

b Consejo Nacional de Investigaciones Científicas y Técnicas (Conicet), Laboratorio de Investigación en Ciencias Humanas (Lich), Buenos Aires, Argentina.
} 
inicial, como a la formación en servicio de quienes se desempeñan bajo diferentes figuras pedagógicas en el sistema educativo. En el marco de esta línea política, en el año 2013 y bajo la Presidencia de Cristina Fernández de Kirchner, se crea el Programa Nacional de Formación Permanente (PNFP), conocido como "Nuestra Escuela".

En su propuesta original, el Programa se orienta a la "formación en ejercicio, gratuita y universal para todos los docentes del país", a lo largo de tres cohortes consecutivas, de tres años cada una, para el sector público y privado. Su propósito se suscribe a la "demanda histórica de formación continua y situada" como constitutiva del trabajo docente desde el nivel inicial al superior no universitario (ARGENTINA, 2015).

El ascenso de Mauricio Macri a la Presidencia, en diciembre de 2015, produjo un profundo giro en las políticas educativas que venían siendo ejecutadas. La suspensión de programas y proyectos socioeducativos y la disolución de equipos de trabajo a nivel nacional y provincial, fueron la consecuencia directa de procesos de desfinanciamiento del sistema educativo. Concretamente el PNFP a partir del 2016 pasó a denominarse Programa Nacional de Formación Situada (PNFS), en el marco de un proceso que entendemos combina dinámicas de descentralización y desconcentración a las provincias de la responsabilidad sobre el sostenimiento del Programa.

En este artículo nos proponemos reconstruir las transformaciones que el PNFP tuvo desde sus orígenes a la actualidad. Centramos el análisis en lo acontecido tras el cambio de gobierno a nivel nacional en diciembre de 2015, tomando como recorte para el estudio escuelas secundarias de modalidad técnica y común de la provincia de Córdoba.

Desde los aportes de la teoría de la regulación social, pretendemos caracterizar las nuevas formas de regulación del programa identificando las estrategias desplegadas por el gobierno central en relación al período 2016-2019; eso involucraría el último año de la primera cohorte (2014-2016), toda la segunda (2015-2017) y toda la tercera (2016-2018). El análisis de los cambios en los procesos de regulación del PNFP al PNFS permite no solo la descripción de la producción de reglas que orientan el funcionamiento del Programa desde las acciones del Estado Nacional, sino también del proceso de acomodación que realiza la provincia de Córdoba en función de esas mismas reglas.

Desde un enfoque de tipo cualitativo, el trabajo de campo se enmarca en dos investigaciones en curso, una focalizada en el análisis de la puesta en acto de políticas para garantizar la Educación Secundaria Obligatoria (ESO) y otra para 
la Educación Técnico Profesional (ETP). Recuperamos principalmente el aporte de entrevistas realizadas a actores estatales que se desempeñan en diferentes niveles de la administración escolar y que poseen diversas tareas en la puesta en acto del Programa.

El escrito se organiza a partir de recuperar conceptos relevantes para indagar las transformaciones en los modos de regulación de las políticas de formación docente en servicio. Seguidamente, se caracteriza el PNFP y el contexto de diseño de esta política. En tercer lugar, presentamos las referencias teóricas y metodológicas de la investigación. Como cuarta instancia, exponemos el análisis de las redefiniciones que el Programa tuvo entre los años 2016-2019 en la provincia de Córdoba, para finalmente plantear algunos avances interpretativos.

\section{Procesos de descentralización y desconcentración en el sistema educativo argentino}

La década de los noventa promovió profundos cambios en los modos de regulación de las políticas educativas en varios países de la región iberoamericana, reformas que se orientaron por valores y criterios economicistas definidos por los Organismos de Financiamiento Internacional (OFI). Las políticas de descentralización se constituyeron en una dimensión constitutiva de la reforma y del nuevo rol que el Estado Nacional debía asumir en el marco de los denominados procesos de reforma estructural. Del Estado Benefactor, garante de derechos, pasamos a un Estado que deviene en regulador de los servicios que fueron descentralizados y/o privatizados (ANGULO RASCO, 1999; GARCÍA DELGADO, 1998). De esta forma, la descentralización habilita la disminución de los servicios sociales prestados por el Estado y la privatización de un gran número de empresas estatales, aliviando el déficit público, al mismo tiempo que aumentando la dependencia con el sector privado.

En Argentina, la descentralización del nivel secundario y superior no universitario llevada a cabo en el marco de la denominada Reforma Educativa de los noventa tendrá como antecedente la descentralización de los niveles de enseñanza inicial y primario en el año 1978, en plena dictadura militar de Jorge Rafael Videla. Bajo la primera presidencia de Carlos Menem, la Reforma Educativa de los noventa se inició con la sanción de la Ley de Transferencia nº 24.049 en 1991 y se completó más adelante con la sanción de la Ley Federal de Educación $\mathrm{n}^{\mathrm{o}}$ 24.195 en 1993, primera ley orgánica de educación que comprendió todos los niveles y jurisdicciones del Sistema Educativo Argentino. De esta manera, la descentralización a través de la transferencia a las provincias fue uno de los compromisos adoptados por el Gobierno Nacional ante el Fondo Monetario 
Internacional en medio de las negociaciones para la refinanciación de la deuda externa (1989-1991) (SENÉN GONZÁLEZ; ARANGO, apud GOROSTIAGA; ACEDO, XIFRA, 2003).

El slogan que graficó al sistema educativo en Argentina con posterioridad a la ley de transferencia fue: "Un ministerio sin escuelas", pues, efectivamente el Ministerio de Educación de la Nación ya no tenía más escuelas a cargo e incluso hacia fines del 2001 se planteó la posibilidad de eliminar el Ministerio de Educación (CLARÍN, 2001), acción resistida por los y las trabajadores y por los gremios docentes. Para Filmus (1996), si bien las lógicas de la descentralización se fundamentaron en este período en la promoción de la mejora en la toma de decisiones, la mejoría en la calidad educativa o la mentada democratización del sistema educativo, la lógica que prevaleció en aquella descentralización fue la económica. Se buscaba reducir el gasto del gobierno central delegando mayor poder y responsabilidades a las provincias.

Como lo refleja la investigación educativa producida en Argentina, en el marco de los cambios introducidos por la Ley Federal de Educación (FELDFEBER; IVANIER, 2003; FILMUS, 1997; MIRANDA; SENEN GONALEZ; LAMFRI, 2003), la descentralización del sistema educativo en Argentina promovió dinámicas de recentralización que operaron por lo menos a través de cuatro instrumentos: la evaluación, por medio de los Operativos Nacionales de Evaluación de la Calidad; la definición del currículo, por medio de los Contenidos Básicos Curriculares (CBC); las políticas compensatorias, cuyo principal emblema fue el Plan Social Educativo (PSE); y la formación docente, por medio de las conformación de la Red Federal de Formación Docente Continua.

En líneas generales, la investigación educativa a nivel mundial y regional (BALL, 1998; BARROSO; AFONSO, 2002; DUSELL, 2005; FEIJOO, 2003; KRAWCZYK; VIEIRA, 2007; MIRANDA, 2005; POPKEWITZ, 1996; TENTI FANFANI, 2003) ha reconocido el abismo que separa el plano discursivo del plano práctico en la puesta en acto de las políticas diseñadas para descentralizar los sistemas educativos. En el contexto portugués, y ante la redefinición en periodos temporales similares al argentino de un nuevo panorama político-administrativo para dicho sistema educativo, Formosinho et al. (2005, p. 25) manifiesta la necesidad de abordar de modo conjunto los procesos de desconcentración y descentralización, ya que los procesos de descentralización son generalmente precedidos de acciones de desconcentración, las cuales comúnmente se confunden o venden la desconcentración por descentralización.

De este modo, la combinación de retóricas descentralizadoras con normativas que responden solo a procesos de desconcentración o de recentralización ponen de manifiesto que la desconcentración no es necesariamente una etapa en el proceso 
de descentralización; todo lo contrario, puede tener por objetivo dificultarla. En articulación con las características que asume la acción estatal descentralizadoradesconcentradora, el accionar del Estado se configura como un Estado que regula las reglas del juego y evalúa los resultados (GARCÍA DELGADO, 1998).

\section{Políticas educativas y formación docente durante el kirchnerismo}

Durante la Presidencia de Néstor Kirchner, la definición de la educación como derecho social y el rol del Estado como garante del mismo se tradujeron en numerosas políticas (FELDFEBER; GLUZ, 2011).

Entre las acciones del gobierno electo se destaca el diseño de un nuevo marco normativo para el sistema educativo, en donde se señala la sanción de la Ley de Garantía del Salario Docente y 180 Días de Clase (2003), la Ley del Fondo Nacional de Incentivo Docente (2004), la Ley de Educación Técnico Profesional (2005), la Ley de Financiamiento Educativo (2005), la Ley Nacional de Educación Sexual Integral (2006) y la Ley de Educación Nacional (2006), identificándose de esta manera -entre otros-, la intención de impulsar la jerarquización y revalorización de la formación docente como factor clave del mejoramiento de la calidad del sistema educativo e incluyéndose líneas de formación en servicio para los docentes (FELDFEBER, 2010). Al respecto, Terigi (2016) señala que

El punto de partida fue una descripción crítica de los problemas del sistema de formación docente (fragmentación y segmentación de la oferta, falta de planificación de las instituciones y las carreras, debilidad de las regulaciones, insuficiente desarrollo pedagógico del currículum de formación docente, entre otros [Ministerio de Educación, 2015b]) y la convicción de que la descentralización de la formación docente y las políticas nacionales de acreditación de instituciones (ambas iniciativas del gobierno de Menem) no habían sido herramientas adecuadas para mejorar la formación (p. 18).

De esta manera, el gobierno electo en 2003 fue generando de manera paulatina, primero con la creación del Instituto Nacional de Formación Docente (Infod) en el 2006 y luego, con más énfasis desde el 2012, cuando incorporó la planificación de la formación docente al segundo Plan Nacional de Educación Obligatoria, las condiciones para articular políticas de formación docente con otras acciones para la optimización del funcionamiento del sistema escolar (TERIGI, 2016). 
Entre las medidas adoptadas se destaca la creación en el año 2013 del Programa "Nuestra Escuela" a través de la Resolución n 201 del Consejo Federal de Educación. Esta denominación recibió el PNFP, el cual nace con el propósito de propiciar la profundización de la formación disciplinar y didáctica de los docentes de todos los niveles, modalidades y gestión, tanto pública como privada. Es destacable que el Programa nace como una iniciativa federal con financiamiento del Estado Nacional en coordinación con cinco sindicatos docentes de representación nacional: Confederación de Trabajadores de la Educación de la República Argentina (Ctera), Unión Docentes Argentinos (Uda), Asociación del Magisterio de Enseñanza Técnica (Amet), Confederación de Educadores Argentinos (Cea) y Sindicato Argentino de Docentes Privados (Sadop).

\subsection{Aspectos generales sobre el PNFP}

El Programa se extendió a lo largo y ancho del país abarcando a la totalidad de las unidades educativas y a todos sus docentes, adquiriendo así un carácter federal. Cada una de las cohortes ( 3 en total, desarrolladas de manera consecutiva) se organizó previendo el desarrollo de siete jornadas institucionales anuales. Además, el programa fue pensado y organizado con base a dos componentes concretos: un componente (I) institucional: centrado en las instituciones educativas, que buscó promover el análisis pedagógico y organizacional de los problemas de enseñanza y los aprendizajes de cada nivel. Se inició en el año 2014 y abarcó alrededor de 40 mil escuelas en todo el país, desarrollándose en jornadas institucionales obligatorias y en ejercicio; como así también otras estrategias complementarias como ateneos didácticos, círculos de directores y talleres para la enseñanza; y otro componente (II) por destinatarios especificos: centrado en las prioridades formativas de actualización y profundización de acuerdo a roles, niveles, disciplinas y modalidades en los que se desempeña cada docente, vinculados con los objetivos del Plan Nacional de Educación Obligatoria y Formación Docente. Asimismo, promovió la creación de 13 postítulos en convenio con 51 universidades, institutos y autoridades provinciales (TERIGI, 2016, p. 19).

Los ejes de formación para cada nivel educativo en los tres años que se estipuló para cada cohorte dan cuenta de los temas centrales previstos en la Resolución CFE n ${ }^{\circ}$ 201/13 del Plan Nacional de Educación Obligatoria y Formación Docente y de las decisiones referidas a temáticas relevantes y prioritarias de la Ley de Educación Nacional. Además, se dota a las escuelas de materiales diversos: cuadernillos impresos organizados en series, videos de diferentes fuentes o específicos, artículos periodísticos, relatos, documentos históricos, etc., de utilidad para el desarrollo de los encuentros. 


\section{Referencias teóricas y metodológicas}

Para poder caracterizar las nuevas formas de regulación del PNFP desplegadas por el gobierno nacional en el período 2016-2019 partimos de considerar a esta política de formación docente, en línea con los planteos de Barroso (2006), no como algo unificado, que impone un modo de hacer, sino como un todo complejo cuya regulación depende de una pluralidad de sujetos que "ponen en acto" las políticas (BALL; MAGUIRE; BRAUN, 2012).

El proceso de regulación de los sistemas educativos implica la producción de reglas que orientan el funcionamiento del sistema y el (re) ajuste de la acción de los sujetos en función de reglas que tienen la función de asegurar el equilibrio, la coherencia y la transformación que cualquier sistema demanda. Concretamente, el concepto de regulación posibilita para Barroso (2006) dos tipos de fenómenos diferenciados e interdependientes: por un lado, una regulación institucional, donde se definen los modos por los cuales son producidas y aplicadas las reglas que orientan el accionar de los actores. Y por el otro lado, una regulación situacional, donde adquieren centralidad los modos por los cuales esos mismos actores se apropian de esas normativas y las transforman. En palabras del autor:

En el primer caso estamos en presencia de [...] el conjunto de acciones decididas y ejecutadas por una instancia (gobierno, jerarquía de una organización) para orientar las acciones y las interacciones de los actores sobre los cuales detentan alguna autoridad (Maroy \& Dupriez, 2000). Esta definición pone en evidencia, en el concepto de regulación, las dimensiones de coordinación, control e influencia ejercida por lo detentores de la autoridad legítima [...] En el segundo caso [...] ella es vista como un proceso activo de producción de reglas de juego (Reynaud, 1997, 2003) que comprende no sólo la definición de reglas (normas, mandatos, restricciones, etc.) que orientan el funcionamiento del sistema, sino también, su (re) ajustamiento provocado por la diversidad de estrategias y acciones de los varios actores, en función de esas mismas reglas [...] (BARROSO, 2006, p. 12-13).

Ambos procesos dan cuenta de la complejidad del proceso de regulación del sistema educativo en donde subyacen una diversidad de fuentes, finalidades, sentidos y lógicas de acción. En este estudio, el concepto de regulación cobra relevancia en tanto nos permite dar cuenta de los cambios en el papel del Estado y de la alteración de sus modos de intervención/financiamiento de las políticas educativas para el PNFP en Córdoba. Nos permite dar cuenta de nuevas formas de regulación impulsadas por el gobierno nacional en el periodo seleccionado. 


\subsection{Aspectos metodológicos}

El trabajo de campo se enmarca en dos investigaciones, que responden a estudios de caso de tipo cualitativo (YIN, 2001): una focalizada en el análisis de la puesta en acto de políticas para garantizar la Educación Secundaria Obligatoria en escuelas de modalidad común; y la otra, en escuelas de Educación Técnico Profesional o escuelas técnicas.

Para reconstruir el proceso de regulación del PNFP al PNFS en Córdoba recuperamos aportes de entrevistas realizadas a actores que se desempeñan en diferentes niveles de la administración escolar y que cumplen distintas tareas de gestión en la puesta en acto del programa en la escuela secundaria: 2 tutoras del PNFS de la región Capital, responsables del trabajo con equipos directivos de 22 escuelas (modalidad común y técnica), y 1 supervisora de la misma región de Educación ${ }^{1}$. La elección de los entrevistados fue realizada al azar, considerando la cantidad de tutores y supervisores por región educativa, siendo este número representativo del total. Asimismo, cabe destacar que ambos cargos, tutor $^{2}$ y supervisor, son cargos asumidos por selección de antecedentes en el primer caso y por concurso público en el segundo, por ello desligados de cualquier filiación política partidaria.

Las entrevistas fueron semi-estructuradas, grabadas con consentimiento de los entrevistados, de aproximadamente 3:00 hs. de duración cada una, buscando indagar a través de la palabra de los participantes indicadores que nos permitan elaborar categorías de análisis para la reconstrucción del proceso de puesta en acto del PNFP al PNFS en la provincia de Córdoba. Como ejes principales se plantearon: tipos de participación en el programa (rol, año de ingreso, etc.); organización y administración del programa (equipos de trabajo, recursos materiales y humanos, entre otros) y experiencias vivenciadas respecto al paso de PNFP al PNFS (cambios/transformaciones/permanencias evidenciadas).

La estrategia de análisis se basó en la reconstrucción del proceso de regulación del programa en el período estudiado, a partir de la articulación entre los discursos de los entrevistados, bibliografía sobre el tema y la documentación existente a nivel

Cabe aclarar que existen 5 subregiones dentro de la Región de la Educación Secundaria Común que involucra a la Capital de la provincia y 3 subregiones de la modalidad de la Educación Técnico Profesional para Capital. Respecto a la Educación Común, cada Tutor tiene a cargo entre 11 y 22 escuelas de capital, mientras que los Tutores de Educación Técnico Profesional tienen entre 12 y 6 escuelas.

2 Debemos aclarar que los tutores no pertenecen formalmente a ninguno de los niveles de gestión de la política y tampoco son parte de las dinámicas escolares, sino que son seleccionados en el marco del Programa por contrato a término. Sin embargo, tienen la capacidad de influir en el territorio desde su quehacer específico en tanto capacitaciones de los equipos directivos y coordinadores de las Jornadas Institucionales en las escuelas. 
nacional y provincial sobre el programa. Para el procesamiento de la información se utilizó Atlas.ti como herramienta informática de apoyo.

\section{El gobierno de la alianza cambiemos}

En el 2015, a partir de la asunción de Mauricio Macri como Presidente, las políticas educativas que venían siendo ejecutadas dieron un giro importante. García Delgado y Gradin (2017) hablan de una etapa caracterizada por una feroz disputa por la reestructuración de la distribución de la riqueza a favor de los sectores concentrados de la economía; por el inicio de un nuevo proceso de endeudamiento externo; la apertura de la economía; la desindustrialización; y el intento deliberado de bajar salarios y niveles de consumo de los sectores mayoritarios.

En el ámbito educativo, se evidencian procesos de desfinanciamiento del sistema ligados principalmente al fuerte incremento de la inflación y a la subejecución de los presupuestos de la cartera educativa. La suspensión de programas y proyectos socioeducativos (como los Centros de Actividades Infantiles y Juveniles), la disolución de equipos de trabajo a nivel nacional y provincial, el desmantelamiento de secretarías y direcciones, la desaparición de los Ministerios de Salud y de Ciencia y Tecnología con los cuales existían proyectos conjuntos y, la redefinición del Programa Conectar Igualdad, se constituyen en manifestaciones evidentes del recorte presupuestario en Educación.

Rodríguez (2017) plantea que las principales modificaciones se dieron en cuatro áreas: políticas socioeducativas, evaluación, formación docente y nuevas tecnologías. En relación a la primera, plantea un achicamiento administrativo y presupuestario y un proceso de descentralización a través del cual se disolvieron la gran mayoría de los equipos del nivel nacional y se transfirieron fondos directamente a las provincias, al tiempo que otros programas socioeducativos se eliminaron o redefinieron sus objetivos. En el ámbito del Infod, se suprimieron acciones que se estaban realizando en relación a los cursos y postítulos que formaban parte del Componente II del PNFP, y se delegó parcialmente ese presupuesto a las provincias. Asimismo, se destaca la jerarquización del área de evaluación durante este gobierno, elevando su rango al nivel de Secretaría y declarándola como política prioritaria.

En este contexto, a partir del 2016, el PNFP pasó a denominarse PNFS en el marco de un proceso de desconcentración donde las provincias fueron garantes del sostenimiento del programa, y también responsable ante el Estado Nacional sobre los resultados. Operativamente, la coordinación del PNFS en Córdoba siguió 
a cargo de la Subsecretaria de Promoción de la Igualdad y Equidad, dependiente del Ministerio de Educación provincial, en tanto nivel de administración central del programa en la jurisdicción. Luego, a un nivel de administración intermedia, se encontrarían los tutores/capacitadores y los supervisores, es decir, el Equipo Técnico Territorial y, por último, en el nivel institucional, los equipos directivos de cada escuela, capacitados en los círculos de directores.

Un estudio coordinado por la Universidad Pedagógica Nacional (Unipe) publicado en noviembre de 2019, da evidencia de un congelamiento del presupuesto destinado a políticas para la formación docente de $\$ 1.400$ millones de pesos, desde el año 2016 con proyección a 2020. Este proceso es definido por los especialistas como "un típico caso de vaciamiento silencioso de una política, basado en mantener su presupuesto nominal en un contexto de alta inflación (más de $200 \%$ en cuatro años)" y en donde el presupuesto real destinado para el sostenimiento del PNFS “pasa de \$ 1.345 millones de pesos en 2016 a \$ 382 millones en 2020" (UNIPE, 2019, p. 16).

Esta tesis encuentra referencial empírico a partir del trabajo de campo que se viene desarrollando en escuelas secundarias. Planteamos que en contexto de ajuste estructural el cambio de denominación del programa: de PNFP a PNFS, supone que lo "situado" avala la estrategia de desconcentración y desfinanciamiento del Componente I, hecho que estaría llevando al "desvanecimiento" total del Programa. A continuación, desarrollamos esta idea a partir de tres categorías teóricas: el proceso de desconcentración del programa, los cambios en los contenidos de formación del programa y la paulatina pérdida de la figura de tutor/capacitador.

\subsection{Hacia la desconcentración del programa: recursos escasos y mecanismos de control centralizados}

Entre los años 2016 y 2019, se efectivizaron cinco instancias de formación por año de las siete previstas originalmente por el programa. Además, datos relevados dan cuenta que, en algunas provincias como Buenos Aires, transitando mediados del año 2016, todavía no habían comenzado con el desarrollo del Programa (CANCELA; FLORES; SERVIA, 2016). De este modo, gremios como Ctera, Sindicato de Trabajadores de la Educación de Buenos Aires (Suteba) y Amet acompañaban el pedido de "normalizar" la ejecución del Programa y "denunciaban" algunas modificaciones en el PNFP desarrolladas de manera unilateral:

Denunciamos que después de meses de parálisis del PNFP, fueron reformulados por la nueva gestión los documentos producidos y sus mecanismos de implementación DE MANERA UNILATERAL. 
De esta manera, mientras se declama la continuidad del PNFP, se contradicen los postulados básicos de la normativa vigente desconociendo y discontinuando la aplicación del Acuerdo Paritario (SUTEBA, 2016).

Como lo menciona Rodríguez (2017), los cambios evidenciados en la dirección de políticas educativas para el sector de la formación docente, tras el cambio de gobierno, se manifiestan en la eliminación de acciones por parte del Infod en torno a cursos y postítulos que formaban parte del Componente II del PNFP y la delegación parcial del presupuesto a los gobernadores. Ello tuvo un efecto evidente a nivel de las provincias: la "ralentización" en el desarrollo del Programa acompañada de la introducción de estrategias de desconcentración del Programa. En este sentido, el cambio de denominación coincide con un proceso de desconcentración a las provincias, el cual en palabras de una supervisora regional queda explicitado de la siguiente manera:

La Subsecretaría de Promoción de la Igualdad y Equidad, dependiente del Ministerio de Educación provincial sigue asumiendo la coordinación del Programa. La plata entendemos que la sigue poniendo Nación, pero cada provincia decide si destina esos recursos para el PNFS o para cualquier otra cosa, puede ser por eso que haya desaparecido en muchas provincias (Supervisora, diciembre 2018).

La transferencia de los fondos a las provincias sin una directiva concreta sobre el destino de los mismos y la paulatina ausencia de elaboración de contenidos para las diferentes instancias de formación del programa (como se verá en el próximo apartado), fueron garantía de un proceso de desconcentración con un claro desfinanciamiento del mismo a través de los años (UNIPE, 2019):

[...] hay parte de la plata que la sigue poniendo Nación, nosotros somos todos monotributistas y le facturamos a la provincia. Nación manda una partida y provincia distribuye, pero no sé cómo [...] Una colega me decía que en otras provincias no estaba funcionando [...] Ahora bien, todos los informes y la rendición de cuentas de lo que se hace en la escuela se manda a Nación a través de una plataforma virtual que tiene provincia, el CIDI ${ }^{3}$ (Tutora 2, PNFS, mayo, 2019).

EI CIDI (Ciudadano Digital) es una plataforma tecnológica que posibilita a los ciudadanos acceder de forma simple, en un único lugar y con una misma cuenta de usuario, a todos los trámites y servicios digitales que brinda el Gobierno de la Provincia de Córdoba. 
El programa siguió siendo descentralizado financieramente a las provincias, pero la Nación siguió manteniendo el control/decisión a nivel central sobre cuestiones técnico-administrativas; ello, en el marco de un agudo proceso hiperinflacionario que en la provincia de Córdoba se pone de manifiesto a través de un paulatino proceso de reducción de los recursos para el sostenimiento del Programa.

\subsection{Cambios en los contenidos: de una acción planificada centralmente a la autonomía institucional de las jurisdicciones}

Como se mencionaba en el Apartado 2.1, en la propuesta original del PNFP el gobierno nacional es garante de la distribución de materiales diversos, cuadernillos impresos en series, videos, artículos, documentos históricos, etc. Sin embargo, estas acciones fueron mermando desde 2016, siendo las provincias las encargadas de empezar a dotar de sentido a las propuestas. Como lo expone una de las tutoras:

Hubo material de Nación que se dejó de hacer. Vos tenías en una página de Nación bloque por bloque el material teórico, con materiales de trabajo, con la planificación hasta 2019. Eso se dejó de actualizar y en cierta forma quedó librado al azar, a lo que cada provincia haga y tomara ese material o no. Córdoba pudo sostener el programa y la estructura. Nosotros tenemos tutores de inicial, de primaria, secundaria, jóvenes y adultos e institutos de formación docente. En otras provincias eso se simplificó o ya no existe (Tutora 1, PNFS, abril, 2019).

La falta de orientaciones por parte de Nación no solo fue haciendo que se desvirtúen los objetivos del Programa, sino que también vaya perdiendo valor simbólico para los destinatarios. Lo situado sin una pauta de organización y direccionalidad para la elección de los contenidos y formatos para las Jornadas Institucionales quedó librado al azar de la decisión de cada provincia y de sus equipos de trabajo; provocando heterogeneidad de propuestas a la vez que el debilitamiento del Programa como garante de una formación universal e igualitaria:

[...] acá tiene 3 componentes: 1- de formación situada en las escuelas; 2- de contenidos, se trabaja creando materiales y cursos y, 3) de monitoreo y seguimiento [...] son temáticas de esta jurisdicción y de políticas provinciales. Hasta el año pasado siempre había un temario por jornada, ahora se trata que cada escuela seleccione dentro de un temario los temas más pertinentes para la escuela. El año pasado (2018) se permitió que cada director elija... (Tutora 1, PNFS, abril, 2019). 
Estas mutaciones en el trabajo con los tutores también han generado un vaciamiento de contenidos y en las formas de trasmitirlos en las Jornada Institucionales en las escuelas, provocando muchas veces el desgano y desinterés en la propuesta tanto por parte de directivos como de docentes:

[...] ahora cada escuela esta con su tema, los directores se sienten muy solos. En general suelen hacer clases expositivas, video, video, trabajo en grupo, puesta en común. Siempre puesta en común y hay veces que no es necesario. Les hacen leer algo y después sólo lo recuperan, cuesta mucho [...] La mayoría de los directores no se quiere mover del video y hasta acortan el tiempo. Todo termina con un afiche, producir para nada...Genera un desgaste tremendo en la institución (Tutora 1, PNFS, abril, 2019).

Todo esto, además, fue llevando a que las horas de trabajo en cada jornada se redujera como decisión de cada directivo y se perdiera un espacio de trabajo que venía siendo muy productivo para las escuelas, en tanto instancia de intercambio y coordinación de decisiones didáctico-pedagógicas para el proceso de enseñanza y aprendizaje.

\section{3 "No sabemos si seguimos": el desvanecimiento de la figura del tutor}

Entre los principales dispositivos del Componente I están las instancias de formación en los Círculo de Equipo Directivos y las Jornadas Institucionales. Estas últimas, a cargo de cada jurisdicción, tienen como objetivo la realización de producciones colectivas para evaluar los avances del proceso formativo, como así también la realización de producciones de integración.

Las jornadas son coordinadas por un Equipo Técnico Territorial (tutores/capacitadores y supervisores) y los equipos directivos escolares, quienes participan de una capacitación previa desarrollada por el órgano central de gobierno. La figura del "tutor" es sustancial para el desarrollo de la propuesta, porque son quienes están a cargo junto con el director escolar de las Jornadas de Trabajo en la escuela.

Desde el principio acá la cantidad de tutores no se modificó, y en general somos docentes de la modalidad, eso es muy importante. Igual es insuficiente sobre todo en secundaria donde cada tutor tiene aproximadamente 25 escuelas [...] Yo creo que este año (2019) ha sido un año como de "aguantemos" a ver que viene, por un lado, en el sentido de qué viene a nivel provincial. Y por el otro, porque Nación no da señales (Tutora 2, PNFS, mayo, 2019). 
A los cambios en el rol del Estado se suman los cambios de gobierno en la provincia producto de las últimas elecciones. Al momento de las entrevistas (abril-mayo 2019), las tutoras aseguraban estar transitando por un momento de "incertidumbre", pues no sabían si el PNFS iba a seguir y si los tutores iban a continuar estando.

En un informe elaborado por la Subsecretaría de Promoción de la Igualdad y la Equidad de la provincia, se destaca que los directores escolares rescatan como virtudes de la figura del tutor: "la planificación de sus tareas, los abordajes teórico prácticos, así como su presencia durante el desarrollo de las Jornadas Institucionales" (CÓRDOBA, 2017, p. 13). En suma, la posible desaparición de la figura del tutor traería entonces aparejada una serie de dificultades ligadas a la sobrecarga de tareas del director escolar, al tener que planificar las Jornadas Institucionales en soledad; asimismo, se perdería el espacio de comunicación que se genera entre el Supervisor y el director escolar a través de la figura del tutor.

\section{Notas finales sobre un programa que parece... no continuará}

[...] Podía asistir a cualquier curso del Ministerio de Educación, desde donde repartían subsidios a grupos privados que ofrecían capacitación con un espíritu más de mercado que pedagógico. Los shoppings se habían puesto de moda y el Ministerio parecía un supermercado de Educación; incluso ya entonces había intentos de convertir en shopping varias escuelas, aprovechando edificios excelentes que conservaban un estilo centenario (PUIGGRÓS, 2015, p. 83).

Los avances en el trabajo de campo muestran que el "noventoso retorno" a las políticas de descentralización se promovió en el último gobierno apelando a la "voluntad" de la provincia de Córdoba para destinar el financiamiento nacional al sostenimiento del PNFS. La voluntad política discursivamente se traduce en autonomía y Córdoba (felizmente), a través de la Subsecretaría de Promoción de la Igualdad y la Calidad, opta por el sostenimiento del Programa asumiendo, además, nuevas tareas que tienen que ver con el desarrollo de contenido para el PNFS. Sin embargo, y ante el panorama que promete el nuevo cambio de gobierno a nivel nacional, el ciclo lectivo 2020 inició para los docentes cordobeses con jornadas de PNFS en el mes de febrero pero sin círculos de directores, ni acompañamiento de los tutores, al tiempo que en los pasillos de las escuelas se escucha "el PNFS se terminó". 
Siguiendo los aportes de Formosinho et al. (2005), planteamos entonces que el principal instrumento del gobierno de la Alianza Cambiemos para reducir la inversión del Estado Nacional fue a partir de una estrategia que combinó descentralización con desconcentración. Si bien el programa desde sus inicios fue planteado a partir de una estrategia descentralizadora, las instancias de formación de los diferentes trayectos eran garantizadas por el Gobierno Nacional fomentando la subsistencia y articulación federal del programa. Sin embargo, estos recursos en el marco de recortes presupuestarios para el sector educativo llevaron a una reducción de recursos para el sostenimiento del Programa. En este sentido, planteamos que se acaba promoviendo dinámicas de desconcentración de PNFS a las provincias.

La desconcentración/desentendimiento del Estado Nacional como garante de la ejecución del Programa habría propiciado el terreno para que Córdoba, en el marco de la autonomía delegada, redefina los contenidos de la formación docente y en contexto de crisis fiscal promueva también la progresiva reducción de recursos financieros y humanos que el funcionamiento del PNFS demanda.

Parece que la experiencia de la década de los noventa y sus efectos en el sistema de formación docente no fue valorada por los hacedores de políticas, o quizás sí, pero para atender a los intereses de quienes siguen creyendo (aún en democracia) que "reducir el Estado es agrandar la Nación"4.

La formación situada sigue siendo una demanda histórica, pero retornamos a la desconcentración y al desfinanciamiento. La desconcentración/desentendimiento/ desfinanciamiento del Estado Nacional en un país tan desigual como Argentina no puede propiciar buenos resultados; eso ya lo sabemos. La desconcentración a las provincias no devino en construcción de "shoppings de la formación docente" (PUIGGRÓS, 2015) como en los noventas; sin embargo, ahora está cada vez más claro que los mecanismos de regulación del sistema educativo son cada vez más complejos, más eufemizados, a veces más políticamente correctos, pero nunca más justos para quienes hacen escuela. La promoción de la creatividad y la innovación pedagógica que el PNFS declama descansa en el trabajo del director, pero sin los recursos prometidos.

Muletilla del Ing. Álvaro Alsogaray, recuperada por Martínez de Hoz, Ministro de Economía entre 1976-1980 (gobierno de facto). 


\title{
O retorno de políticas de desconcentração? Mutações no Programa Nacional de Formação Permanente (PNFP). Córdoba, Argentina
}

\section{Resumo}

Nesse artigo pretendemos reconstruir as transformações do Programa Nacional de Formação Permanente (PNFP) de professores, denominado "Nossa Escola". O foco da análise foram as transformações ocorridas na província de Córdoba, Argentina, após a mudança de governo, em nível nacional, em dezembro de 2015. O trabalho de campo faz parte de duas investigações em andamento realizadas em escolas secundárias de modalidades técnico, profissional e comum. Recuperamos contribuições de entrevistas realizadas com servidores públicos que trabalham em diferentes níveis da administração escolar e que têm tarefas distintas na atuação do programa. A partir das contribuições da Teoria da Regulação Social buscamos caracterizar as novas formas de regulação do programa, identificando as estratégias empregadas pelo governo central e provincial no periodo de 2016-2019.

Palavras-chave: Programa Nacional de Formação Permanente. Formação Situada de Professores. Políticas Educacionais.

\section{The return of deconcentration policies? Mutations in the national Program of Permanent Formation (NPPF). Cordoba, Argentina}

\begin{abstract}
In this article, we propose to reconstruct the transformations of the National Program of Permanent Formation (NPPF) for teachers, called "Our School". We focus the analysis on what happened in the province of Cordoba after the change of government at the national level in December 2015. The fieldwork is part of two ongoing investigations in secondary schools of common and technical modality. We retrieve contributions from interviews carried out with actors who work at different levels of the school administration and who have different tasks in the enactment of the Program. From the contributions of the theory of Social Regulation, we aim to characterize the new forms of regulation of the program by identifying the strategies deployed by the central and provincial government in the period 2016-2019.
\end{abstract}

Keywords: National Program of Permanent Formation. Situated Teacher Education. Educational Policies. 


\section{Referencias}

ANGULO RASCO, J. et al. Escuela pública y sociedad neoliberal. Madrid: Miño y Dávila, 1999

ARGENTINA. Ministerio de Educación. Cuadernos de trabajo: Serie Política Educativa. Módulo I: PNFP. Buenos Aires, 2015.

BALL, S. Big policies/small world: an introduction to internacional perspectives in education policy. Comparative Education, Oxford, v. 34, n. 2, p. 119-130, 1998. https://doi.org/10.1080/03050069828225

BALL, S.; MAGUIRE, M.; BRAUN, A. How schools do policy: policy enactmentes in secondary schools. London: Routledge Falmer, 2012.

BARROSO, J. A regulação das políticas públicas de educação: espaços, dinâmicas e actores. Lisboa: Educa, 2006

BARROSO, J.; AFONSO, N. Sistèmes éducatifs, modes de régulation et d'évaluationscolaire et politiques de luttecontre les inégalités en Anglaterre, Belgique, France, Hongrie et au Portugal. Lisboa: Universidade de Lisboa, 2002

CANCELA, M.; FLORES, K.; SERVIA, G. Programa Nacional de formación permanente: tensiones ocultas en los escenarios escolares. In: JORNADAS DE SOCIOLOGÍA DE LA UNLP, 9., 2016, Ensenada. Buenos Aires: Universidad Nacional de La Plata, 2016. p. 1-14.

CLARÍN. Porqué no se puede eliminar el Ministerio de Educación Nacional: nota de opinión. 19 dic. 2001 Disponíve em: https://www.clarin.com/opinion/ puede-eliminar-ministerio-educacion-nacional_0_HkDz4BLeRFl.html. Acceso en: 6 mar 2020.

CÓRDOBA. Ministerio de Educación. Apuntes de trabajo $N^{o}$ 3: Evaluación de acciones del "Programa Nacional Nuestra Escuela" desde la perspectiva de los participantes. Córdoba: Subsecretaría de Promoción de la Igualdad y la Equidad, 2017. (Eje 3: Monitoreo y seguimiento).

DUSSEL, I. Desigualdades sociales y desigualdades escolares en la Argentina de hoy: algunas reflexiones y propuestas. In: TEDESCO, J. C. (comp.). ¿Cómo superar la desigualdad y la fragmentación del sistema educativo argentino? Buenos Aires: IIPE-UNESCO, 2005. p. 85-115.

FEIJÓO, M. Equidad social y educación en los años '90. Buenos Aires: IIPEUnesco, 2003 
FELDFEBER, M. Las políticas de formación docente. Voces en el Fénix, [s. 1.], n. 3, set. 2010. Disponible en: https://www.vocesenelfenix.com/content/laspol\%C3\%ADticas-de-formaci\%C3\%B3n-docente. Acceso en: 16 out. 2019.

FELDFEBER, M.; GLUZ, N. Las políticas educativas en Argentina: herencias de los '90. Contradicciones y tendencias de "nuevo signo". Educação \& Sociedade, Campinas, v. 32, n. 115, p. 339-356, abr./jun. 2011. https://doi.org/10.1590/S0101-73302011000200006

FELDFEBER, M.; IVANIER, A. La descentralización educativa en Argentina: el proceso de transferencia de las instituciones de formación docente. Revista Mexicana de Investigación Educativa, México, DC, v. 8, n. 18, p. 421-445, maio-ago. 2003.

FILMUS, D. Estado, sociedad y educación en la Argentina de fin de siglo: procesos y desafíos. Buenos Aires: Troquel, 1996.

FILMUS, D. La descentralización educativa en Argentina: elementos para el análisis de un proceso abierto. In: COLOQUIO REGIONAL SOBRE DESCENTRALIZACIÓN, 1997, San Jose. Costa Rica: Ministerio de Planificación Nacional y Política Económica, 1997. p. 150-172.

FORMOSINHO, J. et al. Administração da educação: lógicas burocráticas e lógicas de mediação. Porto: Edições ASA, 2005.

GARCÍA DELGADO, D. Estado-nación y globalización: fortalezas y debilidades en el umbral del tercer milenio. Buenos Aires: Ariel, 1998.

GARCÍA DELGADO, D.; GRADIN, A. (comp.). El neoliberalismo tardio: teoría y praxis. Buenos Aires: Flacso, 2017. (Documentos de trabajo, n. 5).

GOROSTIAGA, J.; ACEDO, C.; XIFRA, S. E. Secondary education in argentina during the 1990s. Archivos Analíticos de Politicas Educativas, Tempe, v. 11, n. 17, p. 1-28, May 2003. https://doi.org/10.14507/epaa.v11n17.2003

KRAWCZYK, N.; VIEIRA, V. Homogeneidad y heterogeneidad: un estudio comparativo sobre la reforma educativa de la década del 90 en Argentina, Brasil, Chile y México. Estudios Pedagógicos, Valdivia, v. 33, n. 2, p. 59-80, 2007. https://doi.org/10.4067/S0718-07052007000200004

MIRANDA, E. Decentralization, new form of governance of the educational system and teachers'work in Argentina: from the State to the market? In: ESRC SEMINAR 'PRIVATISATION, NEW FORMS OF GOVERNANCE AND WORK', 7., 2005, London. London: University of London, 2005. p. 1-25. 
MIRANDA, E. M.; SENEN GONZÁLEZ, S.; LAMFRI, N. Z. Políticas de reforma del sistema educativo en los noventa: nuevas configuraciones emergentes a partir de la Ley Federal de Educación y su implementación en Córdoba. Córdoba: Brujas, 2003.

POPKEWITZ, T. Rethinking decentralization and state/civil society distinctions: the state as a problematic of governing. Journal of Education Policy, [s. 1.], v. 11, n. 1, p. 27-51, sep. 1996. https://doi.org/10.1080/0268093960110102

PUIGGRÓS, A. Rosarito, un policial pedagógico. Buenos Aires: Colihue, 2015.

RODRÍGUEZ, L. G. Cambiemos: la política educativa del macrismo. Question, [s. 1.], v. 1, n. 53, p. 89-108, ene./mar. 2017.

SUTEBA ; El Ministerio no cumple lo que declama! 21 out. 2016. Disponible en: https://www.suteba.org.ar/el-ministerio-no-cumple-lo-que-declama-15742. html. Acceso: 20 ene. 2016.

TENTI FANFANI, E. Educación media para todos: los desafíos de la democratización del acceso. Buenos Aires: OSDE-IIPE-Unesco, 2003.

TERIGI, F. Políticas públicas en Educación tras doce años de gobierno de Néstor Kirchner y Cristina Fernández. Análisis, [s. 1.], n. 16, p. 1-41, dic. 2016.

UNIPE. En caída libre: balance del Presupuesto Educativo Nacional durante el Gobierno de Cambiemos. Buenos Aires, 2019.

YIN, R. Estudo de casos: planejamento e métodos. Porto Alegre: Bookman, 2001.

\section{Información de las autoras}

Yanina Débora Maturo: Doctora en Ciencias de la Educación, Universidad Nacional de Córdoba. Docente regular de la cátedra de Política Educacional y Legislación Escolar, Universidad Nacional de Córdoba. Contacto: yanina.maturo@unc.edu.ar

(iD) https://orcid.org/0000-0002-9942-6364

María Cecilia Bocchio: Doctora en Educación. Doctorado en Política y Administración Educativa. Universidad de Lisboa, Becaria Erasmus-Mundus Unión Europea. Contacto: mcbocchio@gmail.com

iD https://orcid.org/0000-0003-3915-6857 